\title{
Colo-Pro: a pilot randomised controlled trial to compare standard bolus-dosed cefuroxime prophylaxis to bolus-continuous infusion-dosed cefuroxime prophylaxis for the prevention of infections after colorectal surgery
}

\author{
Andrew Kirby ${ }^{1,2}$ (D) Eduardo Asín-Prieto ${ }^{3,4}$ • Flora Agnes Burns ${ }^{1}$ • Duncan Ewin ${ }^{2} \cdot$ Kavi Fatania $^{1} \cdot$ Mithun Kailavasan $^{1}$. \\ Saira Nisar ${ }^{1}$ • Agamemnon Pericleous ${ }^{1}$ • Iñaki F. Trocóniz ${ }^{3,4}$ • Dermot Burke ${ }^{1,2}$
}

Received: 6 August 2018 / Accepted: 13 November 2018 / Published online: 5 December 2018

(C) The Author(s) 2018

\begin{abstract}
Standard bolus-dosed antibiotic prophylaxis may not inhibit growth of antibiotic resistant colonic bacteria, a cause of SSIs after colorectal surgery. An alternative strategy is continuous administration of antibiotic throughout surgery, maintaining concentrations of antibiotics that inhibit growth of resistant bacteria. This study is a pilot comparing bolus-continuous infusion with bolus-dosed cefuroxime prophylaxis in colorectal surgery. This is a pilot randomised controlled trial in which participants received cefuroxime bolus-infusion (intervention arm) targeting free serum cefuroxime concentrations of $64 \mathrm{mg} / \mathrm{L}$, or $1.5 \mathrm{~g}$ cefuroxime as a bolus dose four-hourly (standard arm). Patients in both arms received metronidazole (500 mg intravenously). Eligible participants were adults undergoing colorectal surgery expected to last for over $2 \mathrm{~h}$. Results were analysed on an intention-to-treat basis. The study was successfully piloted, with $46 \%(90 / 196)$ of eligible patients recruited and $89 \%(80 / 90)$ of participants completing all components of the protocol. A trialled bolus-continuous dosing regimen was successful in maintaining free serum cefuroxime concentrations of $64 \mathrm{mg} / \mathrm{L}$. No serious adverse reactions were identified. Rates of SSIs (superficial and deep SSIs) were lower in the intervention arm than the standard treatment arm $(24 \%(10 / 42)$ vs. $30 \%(13 / 43))$, as were infection within 30 days of operation $(41 \%(17 / 43)$ vs $51 \%(22 / 43))$ and urinary tract infections $(2 \%(1 / 42)$ vs. 9\% (4/43)). These infection rates can be used to power future clinical trials. This study demonstrates the feasibility of cefuroxime bolus-continuous infusion of antibiotic prophylaxis trials, and provides safety data for infusions targeting free serum cefuroxime concentrations of $64 \mathrm{mg} / \mathrm{L}$. Trial registration: NCT02445859.
\end{abstract}

Keywords Antibiotic $\cdot$ Bolus $\cdot$ Cefuroxime $\cdot$ Colorectal $\cdot$ Continuous, infusion $\cdot$ Prophylaxis

Electronic supplementary material The online version of this article (https://doi.org/10.1007/s10096-018-3435-z) contains supplementary material, which is available to authorized users.

Andrew Kirby

a.kirby@leeds.ac.uk

1 Old Medical School, Leeds General Infirmary, Leeds Teaching Hospitals NHS Trust, Leeds LS1 3EX, UK

2 University of Leeds, Leeds LS3 1EX, UK

3 Pharmacometrics and Systems Pharmacology, School of Pharmacy and Nutrition, University of Navarra, Irunlarrea, 1,

31008 Pamplona, Spain

4 IdiSNA, Navarra Institute for Health Research, Irunlarrea, 3, 31008 Pamplona, Spain

\section{Introduction}

Colorectal surgery is a common procedure, with approximately 100,000 operations annually within England, with 18-27\% developing a surgical site infection (SSI) [1-6]. SSIs are a major healthcare concern as they are associated with increased morbidity, mortality, and cost [7]. In an attempt to prevent superficial and deep SSIs, antibiotic prophylaxis is given peri-operatively, normally as a bolus dose within the hour before surgery. Antibiotic prophylaxis is effective; when initially introduced for colorectal surgery, it reduced superficial and deep SSIs rates from 40 to $10 \%$ [8]. Recent data, however, indicate that SSI rates have increased [2-6, 8]. A potential reason for increased SSI rates may be suboptimal dosing of standard 
antibiotic prophylaxis, secondary to increasing rates of obesity and growing antimicrobial resistance $[9,10]$. In patients undergoing colorectal surgery, one study identified that $20 \%$ of patients were colonised with antibiotic resistant Enterobacteriaceae, a genus associated with approximately $80 \%$ of SSIs after colorectal surgery [11]. Furthermore, standard bolus dosing has been reported as not achieving concentrations believed to be effective for preventing SSIs in operations lasting longer than $2 \mathrm{~h} \mathrm{[12].} \mathrm{A} \mathrm{potential} \mathrm{way} \mathrm{of} \mathrm{improv-}$ ing the effectiveness of prophylaxis is by targeting antibiotic concentrations throughout an operation at concentrations more likely to inhibit the growth of bacteria classified as resistant, by using a bolus dose of antibiotic, followed by a continuous infusion during surgery. We therefore undertook a pilot trial (Colo-Pro) of antibiotic prophylaxis administered as a bolus-continuous infusion, targeting a serum concentration able to inhibit both susceptible and resistant Enterobacteriaceae. The aims of this study were (1) to determine the feasibility of recruiting and following-up colorectal patients in a trial comparing bolus vs. bolus-continuous infusion of prophylactic cefuroxime, and (2) to determine if a bolus-continuous dosing regimen could achieve targeted serum levels of antibiotic through operations without serious adverse reactions, and (3) to describe outcome rates in the recruited study population.

\section{Materials and methods}

Design A pilot, randomised controlled, parallel, singleblinded, single-centre, phase II/III trial was conducted in which patients were allocated in a 1:1 ratio to receive either cefuroxime bolus-infusion or standard bolus-dose antibiotic prophylaxis before colorectal surgery. This was an external pilot trial. All participants also received $500 \mathrm{mg}$ of intravenous metronidazole. Ethical approval was obtained from the Health Research Authority (Reference 15/YH/0260), the protocol was reviewed by the Medical Health Research Authority (MHRA), and the study was conducted according to Good Clinical Practice standards with all patients providing informed consent. The trial was registered at Clinical trials. gov: NCT02445859.

Participants Patients were eligible if they were adults $(\geq$ 18 years) undergoing colorectal surgery (incision, excision, or anastomosis of the large bowel, including anastomosis of small to large bowel) expected to last for more than $2 \mathrm{~h}$. Patients were excluded if they were pregnant, had a cephalosporin allergy or penicillin hypersensitivity, coumarin treatment, concurrent use of probenecid, or a creatinine clearance below $40 \mathrm{~mL} / \mathrm{min}$. Participants were recruited from Leeds Teaching Hospitals NHS Trust from August 2015 to April 2017. Participants were identified and consented on their day of surgery on admitting surgical wards. Participants were enrolled and allocated to their intervention by research doctors (FAB, AP, MK, KF, SN).

Randomisation Randomisation was carried out by generating two lists of random numbers using an online sequence generator (https://www.random.org/sequences/). A person not involved in the trial produced sealed envelopes containing randomisation assignments. Patients were randomised prior to surgery. Patients were blind to the allocation; however, allocation information was known to the surgeon and anaesthetist. Research doctors completed follow-up after randomisation and were not blinded. Patients were randomised in theatres. An operating surgeon confirmed that surgery should be completed prior to the patient being randomised. Recruitment ended when 90 patients had been randomised.

Standard dosing Intravenous (IV) cefuroxime $1.5 \mathrm{~g}$ bolus administered four-hourly throughout surgery. The first dose was given within $1 \mathrm{~h}$ of surgery.

Intervention dosing Cefuroxime bolus-continuous dosing was based on targeting non-protein bound (free) serum concentrations of antibiotic at $64 \mathrm{mg} / \mathrm{L}$ throughout surgery. This was intended to ensure all patients achieved and maintained serum concentrations of at least $4 \times 16 \mathrm{mg} / \mathrm{L}$ throughout surgery. This concentration, $16 \mathrm{mg} / \mathrm{L}$, has been reported as the MIC90 (minimum inhibitory concentration 90 (the lowest concentration of antibiotic at which $90 \%$ of a population growth is inhibited)) for Enterobacteriaceae based on a clinical study of patients undergoing colorectal surgery at Leeds Teaching Hospitals [11]. Achieving $4 \times$ the MIC has been associated with maximal antimicrobial efficacy [13]. Two regimens to achieve these concentrations were evaluated. One regimen was formula-based (non-compartment model) and the second was a population pharmacokinetic twocompartment model (compartment model). Both models used pharmacokinetic parameter estimates identified previously] $[12,14]$. As a safety measure, to limit overall cefuroxime exposure, no more than $6 \mathrm{~h}$ of continuous infusion was administered. In cases where the duration of surgery was longer than $6 \mathrm{~h}$, the infusion was stopped and the dosing regimen reverted to a four-hourly bolus dose from hour 10 of surgery. The loading dose was administered within the hour before surgery, with initiation of the continuous infusion before surgery. Further details of intervention dosing regimens are provided in Online Resource 1.

Data collection and management Creatinine clearance was estimated for each patient using the Cockcroft-Gault equation [15]. Data such as co-morbidities, dose and timing of cefuroxime administration, timing of blood sample collection, incision, and closure time were recorded prospectively. 
Sample size calculation A formal power calculation was not required as this was a pilot study. However, to assess the feasibility of the trial design and to obtain 60 patients with analysable pharmacokinetic data, a recruitment target of 90 patients was set, assuming blood samples were collected from two thirds of patients [16].

Outcomes The main outcomes in this trial are related to trial feasibility. The main outcomes assessed are therefore recruitment rates, adherence to allocated interventions, and protocol completion rates. Protocol violations will therefore be reported and described.

For the purposes of facilitating estimations of clinical outcomes in a subsequent phase three trial, clinical outcomes were determined. Clinical outcomes measured were the rate of Surgical Site Infection (SSI), with superficial and deep SSIs included, within 30 days of operation. SSI definitions were the Centre for Disease Control's (CDCs) National Nosocomial Infections Surveillance (NNIS) criteria for defining SSIs [17, 18]. SSIs were formally assessed on approximately day 5 and day 30 post-operatively by the research team. These assessments were carried out in person when patients were inpatients and by telephone using a structured questionnaire when an outpatient [18]. All in-patient infections, organ space SSIs, urinary tract infections, and Clostridium difficile infections within 30 days of operation (according to standard definitions [19]) were recorded. An outcome of infection within 30 days of surgery was assigned if a patient had any i.e. one or more, infections diagnosed in this time period. Additionally, days of hospitalisation within 30 days of operation and mortality after operation at 30 days and 1 year were recorded. Serious adverse reactions were defined as an event that was serious and believed with reasonable probability to be due to the trial treatment.

Data analysis Data were assessed by an intention-to-treat analysis; patients with all outcome data missing were excluded from analysis. In patients with incomplete outcome data, the last observation was carried forward [20]. Additional analyses included characterisation of antibiotic resistant Enterobacteriaceae colonising the colon pre-operatively, and a pharmacokinetic analysis of intra-operative serum cefuroxime concentrations. The CONSORT 2010 statement: extension to randomised pilot and feasibility trials was considered in the analysis of the data; see Online Resource 2 [21].

Microbiology sample processing Antibiotic effectiveness is related to antibiotic concentrations in patients relative to the susceptibility of bacteria. To determine the susceptibility of colonic Enterobacteriaceae, which we considered to be the most likely cause of post-operative SSIs, rectal or stoma swabs were collected from patients pre-operatively [18]. Swabs were screened for Enterobacteriaceae resistance by two methods: the first isolated the Enterobacteriaceae strain that was numerically predominant, and the second isolated the most antibiotic resistant strain. The predominant strain was identified by inoculating a CLED agar plate with the swab, streaking for isolated colonies, and determining the minimum inhibitory concentration (MIC) of these colonies from the terminal streak. To identify the most resistant organism, a CLED agar plate was swabbed for confluent growth and a $30-\mu \mathrm{g}$ cefuroxime antibiotic disc (Oxoid) was placed in the centre. The growth closest to the disc was cultured to purity, and MICs were determined. MALDI-TOF and a 0.16 to $256 \mathrm{mg} /$ L cefuroxime gradient strip (biomerieux) were used to determine species and MICs respectively. Resistance was defined as cefuroxime MIC > $8 \mathrm{mg} / \mathrm{L} \mathrm{[22].}$

Antibiotic serum concentration processing Blood samples were collected intra-operatively, up to four samples per patient throughout surgery, either via venepuncture or via an intravascular catheter. Blood samples were stored on ice intra-operatively, then, after centrifugation at $3000 \mathrm{rpm}$ for $10 \mathrm{~min}$ at $5{ }^{\circ} \mathrm{C}$. Serum was stored at $70{ }^{\circ} \mathrm{C}$ until testing. Total serum cefuroxime concentrations were measured by high-performance liquid chromatography, which was performed on a Hypersil 5ODS column (HPLC Technology Ltd., Macclesfield, UK) using a mobile phase of methanol:water:phosphoric acid (25:74:1) and with detection by UV absorbance at $254 \mathrm{~nm}$ [23]. Samples were diluted 1:1 with acetonitrile, centrifuged at $5000 \mathrm{~g}$ and a volume of $10 \mu \mathrm{L}$ of the supernatant injected into the chromatograph. Quantification was by the external standard method with intra- and inter-assay precision $(\mathrm{CV})$ below $5 \%$.

\section{Results}

Recruitment From August 2015 to April 2017 of 262 patients screened for study entry, 196 were eligible and 90 provided consent. This gave a recruitment rate of $46 \%$. Of the 90 patients providing consent, 45 were randomised to the standard treatment group and 45 to the intervention group.

Protocol adherence There were 10 protocol violations: In the standard treatment arm, after randomisation, two patients did not undergo eligible colorectal surgery. In the intervention treatment arm, after randomisation, three patients did not undergo eligible colorectal surgery and two did not receive the allocated intervention. Three patients in the intervention arm received eligible colorectal surgery, the allocated intervention, and hospital in-patient follow-up, but did not complete all components of 30-day follow-up. See Online Resource 3 for a patient flow diagram. Of the 40 patients who received the 
intervention dosing, 18 received the non-compartment model dosing and 22 the compartment model dosing.

Baseline characteristics of randomised groups Both standard treatment and intervention groups had comparable baseline characteristics including: age (59 vs. 61 years), weight (78 vs $79 \mathrm{~kg}$ ), rectal resection rate (54 vs $52 \%$ ), ASA score of $\geq$ 3 (16 vs. $21 \%$ ), NNIS (National Nosocomial Infections Surveillance risk index) scores (NNIS $=1,60 \%$ vs. $57 \%$ ), and operation types (open surgery, $30 \%$ vs $29 \%$ ) (Table 1).

Clinical outcomes Surgical site infection was found to be present in $30 \%(13 / 43)$ of standard dosing treatment patients and 24\% (10/42) of patients receiving the intervention dosing treatment. Infection within 30 days of surgery was detected in $51 \%$ of patients in the standard dosing group, and in $41 \%$ of the intervention dosing group. There was only one death, with no deaths in either group within the 30 days after operation. Organ space infections occurred in $5 \%(2 / 43)$ of patients in the standard treatment dosing group and in $14 \%(6 / 42)$ of the intervention dosing group. Urinary tract infections occurred less commonly in the intervention group at $2 \%$ (1/42) compared to the standard treatment group at $9 \%$ (4/43). Outcomes are presented in Table 2.

Adverse reactions No serious adverse reactions were identified; specifically, no cases of Clostridium difficile infection were identified within 30 days of surgery.

Pharmacokinetics A total of 58 patients had intra-operative blood samples collected. Based on a protein binding of $40 \%$, free serum cefuroxime concentrations were determined [12]. These demonstrate that targeted concentrations of $64 \mathrm{mg} / \mathrm{L}$ throughout surgical procedures were achieved by the compartment-based model intervention regimen in 11/13
Table 1 Summary of patient characteristics for per protocol analysable patients

\begin{tabular}{|c|c|c|c|}
\hline & & Standard dosing $(n=43)$ & Intervention dosing $(n=42)$ \\
\hline Sex & Male & $56 \%(24 / 43)$ & $64 \%(27 / 42)$ \\
\hline Age & Mean, years & 59 & 61 \\
\hline Weight & Mean, kg & 78 & 78 \\
\hline \multirow[t]{4}{*}{ Indication for surgery } & Cancer & $72 \%(31 / 43)$ & $79 \%(33 / 42)$ \\
\hline & Crohn's & $14 \%(6 / 43)$ & $7 \%(3 / 42)$ \\
\hline & Ulcerative colitis & $9 \%(4 / 45)$ & $14 \%(6 / 42)$ \\
\hline & Other & $9 \%(4 / 45)$ & $7 \%(3 / 42)$ \\
\hline Rectal resection & Yes & $54 \%(23 / 43)$ & $52 \%(22 / 42)$ \\
\hline $\begin{array}{l}\text { Chemotherapy within } \\
12 \text { months of surgery }\end{array}$ & Yes & $23 \%(10 / 43)$ & $21 \%(9 / 42)$ \\
\hline $\begin{array}{l}\text { Radiotherapy within } \\
12 \text { months of surgery }\end{array}$ & Yes & $26 \%(11 / 43)$ & $24 \%(10 / 42)$ \\
\hline Bowel preparation $^{\mathrm{a}}$ & Yes & $42 \%(18 / 43)$ & $31 \%(13 / 42)$ \\
\hline ASA $3 / 4 / 5^{b}$ & Yes & $16 \%(7 / 43)$ & $21 \%(9 / 42)$ \\
\hline Operation over $3 \mathrm{~h}$ & Yes & $58 \%(25 / 43)$ & $52 \%(22 / 42)$ \\
\hline Charlson score & Mean & 2.1 & 2.7 \\
\hline Wound classification & Clean contaminated & $100 \%(43 / 43)$ & $98 \%(41 / 42)$ \\
\hline \multirow[t]{4}{*}{ NNIS ${ }^{\mathrm{c}}$} & 0 & $33 \%(14 / 43)$ & $33 \%(14 / 42)$ \\
\hline & 1 & $61 \%(26 / 43)$ & $57 \%(24 / 42)$ \\
\hline & 2 & $7 \%(3 / 43)$ & $5 \%(2 / 42)$ \\
\hline & 3 & 0 & $5 \%(2 / 42)$ \\
\hline Stoma formed & Yes & $47 \%(20 / 43)$ & $31 \%(13 / 42)$ \\
\hline \multirow[t]{4}{*}{ Surgical type } & Laparoscopic & $56 \%(24 / 43)$ & $64 \%(27 / 42)$ \\
\hline & Open & $30 \%(13 / 43)$ & $29 \%(12 / 42)$ \\
\hline & Robotic & $7 \%(3 / 43)$ & $2 \%(1 / 42)$ \\
\hline & Lap to open & $7 \%(3 / 43)$ & $5 \%(2 / 42)$ \\
\hline Drain inserted & Yes & $44 \%(19 / 43)$ & $43 \%(18 / 42)$ \\
\hline
\end{tabular}

${ }^{\text {a }}$ Pre-operative bowel preparation $=$ oral formulations or rectal enema

${ }^{\mathrm{b}}$ ASA = American Society of Anaesthesiologists physical status classification

${ }^{\mathrm{c}}$ NNIS $=$ National Nosocomial Infections Surveillance [NNIS] basic SSI risk index 
Table 2 Outcome measures associated with intervention and standard dosing regimens per protocol analysis

\begin{tabular}{lll}
\hline & Standard dosing $(n=43)$ & Intervention dosing $(n=42)$ \\
\hline SSI-S ${ }^{\text {a }}$ within 30 days of surgery & $30 \%(13 / 43)$ & $24 \%(10 / 42)$ \\
Infection within 30 days of surgery & $51 \%(22 / 43)$ & $41 \%(17 / 43)$ \\
Mean length of stay (days) & 9.9 & 9.8 \\
Death within 30 days of surgery & $0 \%(0 / 43)$ & $0 \%(0 / 42)$ \\
Death within 365 days of surgery & $2 \% 1 / 43$ & $0 \% 0 / 42)$ \\
SSI-O $^{b}$ within 30 days of surgery & $5 \%(2 / 43)$ & $14 \%(6 / 42)$ \\
$\mathrm{UTI}^{\mathrm{c}}$ within 30 days of surgery & $9 \%(4 / 43)$ & $2 \%(1 / 42)$ \\
\hline
\end{tabular}

${ }^{\text {a }}$ SSI-S: superficial surgical site infection

${ }^{\mathrm{b}}$ SSI-O: organ space surgical site infection

${ }^{\mathrm{c}}$ UTI: urinary tract infection
(85\%) of patients. The non-compartment model maintained higher concentrations than standard bolus dosing (Fig. 1) but did not achieve targeted concentrations throughout surgery in any studied patients $(0 / 10)$.

Antibiotic susceptibility testing The MIC was different depending upon the susceptibility test performed, with the screen for more antibiotic resistant bacteria identifying bacteria with a higher MIC50 (2 vs $1.5 \mathrm{mg} / \mathrm{L}$ ) and higher MIC90 ( 12 vs $3 \mathrm{mg} / \mathrm{L}$ ), see Table 3 . There was no identified relationship between MIC and outcome.

\section{Discussion}

We have successfully demonstrated the feasibility of performing a trial of cefuroxime bolus-continuous infusion versus standard bolus cefuroxime antibiotic prophylaxis. This conclusion is based on a recruitment rate of $46 \%$, which we consider good due to the complex and multidisciplinary setting in which recruitment was completed. In this trial, as the treatment was finished by the end of a patient's surgical procedure, there were no issues related to adherence. Randomisation was successfully completed, and there were only a limited number of patients whose surgical procedures
Fig. 1 Free serum cefuroxime concentrations for 58 patients according to intervention treatment (compartment and noncompartment) and standard treatment dosing regimens. Horizontal lines represent $64 \mathrm{mg} /$ $\mathrm{L}$ and $16 \mathrm{mg} / \mathrm{L}$

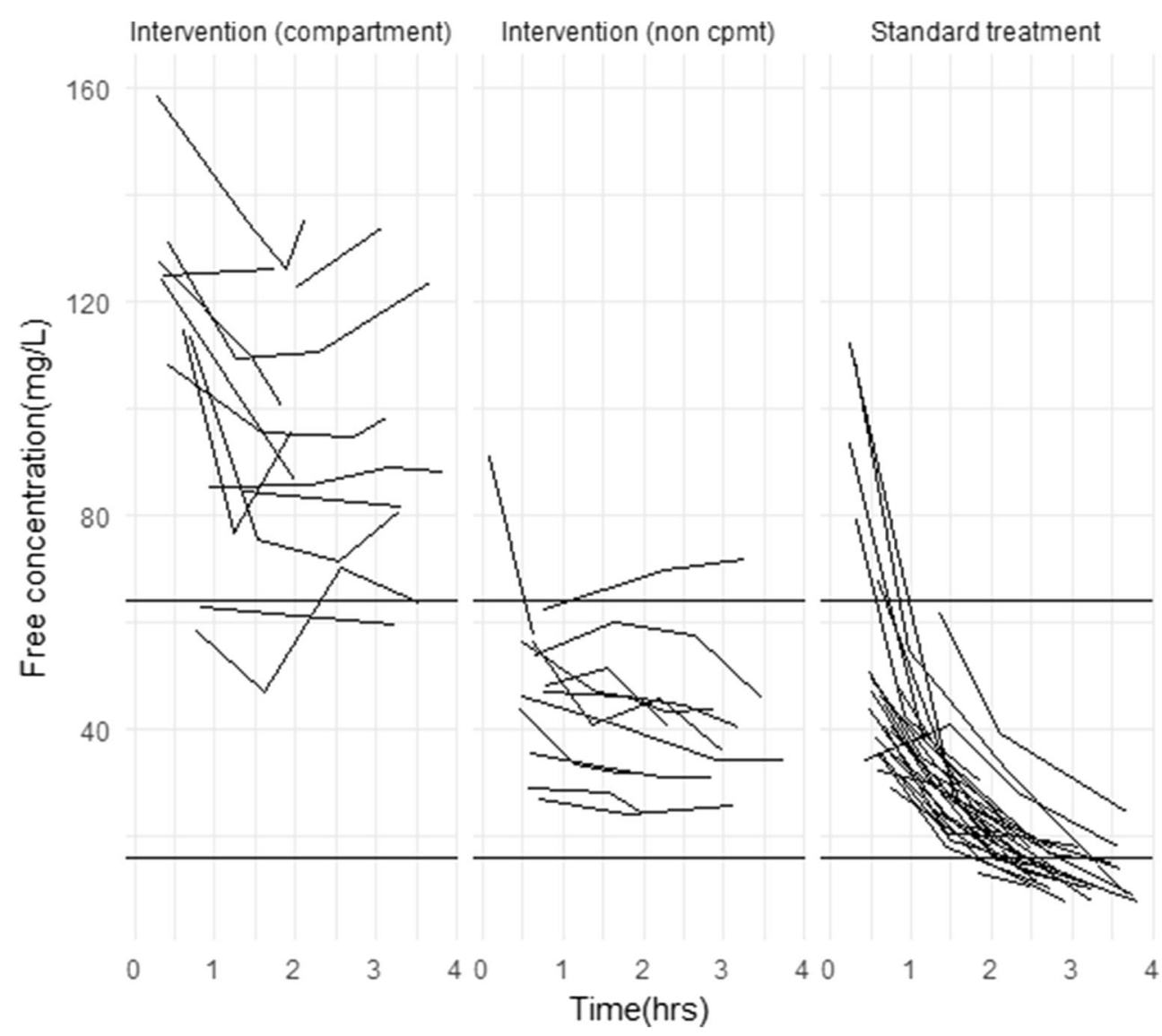


Table 3 Microbiology and antibiotic susceptibility testing results from pre-operative colonic samples

\begin{tabular}{|c|c|c|c|c|c|c|c|c|}
\hline & \multicolumn{4}{|c|}{ Bacterial species identified } & \multicolumn{4}{|c|}{ Susceptibility to cefuroxime (mg/L) } \\
\hline & E. coli & Klebsiella spp. & Other Enterobacteriaceae & None & MIC50 & MIC90 & $\%$ resistant $^{\mathrm{a}}$ & Geometric mean \\
\hline Predominant & $67 / 90$ & $6 / 90$ & $8 / 90$ & $9 / 90$ & 1.5 & 3 & $3 \%(3 / 90)$ & $2.1(n=81)$ \\
\hline Resistant & $52 / 90$ & $9 / 90$ & $19 / 90$ & $11 / 90$ & 2 & 12 & $11 \%(10 / 90)$ & $3.5(n=80)$ \\
\hline
\end{tabular}

${ }^{\text {a }}$ Susceptibility defined as a cefuroxime breakpoint of $>8 \mathrm{mg} / \mathrm{L}$

were not carried out. Future studies should aim to randomise at the last available opportunity, and confirm surgical procedures with the most senior member of the surgical team. The single blinding used in the trial was successfully piloted, but future studies should aim to also blind the patients' clinical teams as well as outcome assessors. There were occasions when a safety concern in relation to low body weight and reduced renal function resulted in non-allocated antibiotic prophylaxis being administered. Guidance within the protocol related to these specific issues could prevent these protocol violations.

In assessing the intervention bolus-continuous dosing regimens, the compartment model-based dosing regimen, which was adjusted for renal function, was the more effective model and achieved target concentrations in $>80 \%$ of patients as predicted. This compartment based-dosing regimen is therefore suitable to be used in a clinical trial targeting free serum concentrations of $64 \mathrm{~m} / \mathrm{L}$ of cefuroxime. The demonstrated feasibility of this trial is important, as it has been shown that antibiotic concentrations at both the start and the end of surgery are important predictors of clinical effectiveness [24]. The bolus-continuous infusion approach to prophylaxis is a strategy that can achieve desired concentrations of antibiotic throughout surgery, including the end of surgery, across multiple surgical procedures, and for multiple antibiotics, making the bolus-continuous infusion approach highly generalisable.

Rates of SSIs (superficial and deep SSIs) were lower in the intervention group, as were any infections and rates of urinary tract infection. These lower rates are consistent with the aim of the intervention, which is to reduce post-operative infections. As the difference in any infection between intervention groups was higher than the difference between SSIs, infection within 30 days of operation may be considered as the optimal outcome measure in future trials. This approach is supported by previous data that show that antibiotics with a long half-life e.g. ceftriaxone, which act like a continuous infusion of a short half-life antibiotic e.g. cefuroxime, reduce post-operative respiratory tract, surgical site, and urinary tract infections [25]. However, these long half-life antibiotics are currently avoided in clinical practice over concerns relating to increased risks of Clostridium difficile infection. All three patients lost-to follow-up at day 30 had an infection identified before their day 30 review. A future study could therefore consider using an intention-to-treat analysis with "the last observation carried forward" method to deal with this as done in this study analysis, or could consider including an outcome measure assessment after a shorter time period e.g. 14 days.

The rate of organ space surgical site infection was higher in the intervention group. Considering known rates of organ space infection it is likely this reflects a lower than expected rate of these events in the standard treatment group [2]. Alternatively, it has been suggested that antibiotics have the potential to select for bacteria with collagenase activity, with the ability to cause anastomotic leak [26]. Higher doses of antibiotic associated with the intervention treatment could therefore be consistent with increased selective pressure on the bacteria, and so increased anastomotic leak and subsequent organ space infection. No mortality was seen in the 30 days after the intervention in either treatment group.

The compartment model intervention was successful in obtaining serum concentrations targeted at $64 \mathrm{mg} / \mathrm{L}$ for the duration of surgery. This study was performed on a population of patients with high rates of cefuroxime susceptible Enterobacteriaceae colonisation of the colon, with resistance detected in $3-11 \%$ of patients, depending on the method of resistance detection used. It is possible that the effectiveness of the intervention may be dependent upon the rate/ characteristics of Enterobacteriaceae resistance.

In summary, completion of this feasibility study suggests that a large pragmatic trial into bolus-continuous infusion of cefuroxime prophylaxis can be completed. As rates of antibiotic resistance increase the need for such trials is becoming more urgent.

Acknowledgements The staff at the Antimicrobial Reference Laboratory in the Bristol Centre for Antimicrobial Research and Evaluation, UK, undertook all serum antimicrobial assays.

Funding The Department of Microbiology, Leeds Teaching Hospitals Charitable Account funded serum antibiotic assays.

Data availability The datasets generated during and analysed during the current study are available from the corresponding author on reasonable request.

\section{Compliance with ethical standards}

Conflict of interest The authors declare that they have no conflict of interest. 
Ethical approval All procedures performed in studies involving human participants were in accordance with the ethical standards of the institutional and/or national research committee and with the 1964 Helsinki declaration and its later amendments or comparable ethical standards.

Informed consent Informed consent was obtained from all individual participants included in the study.

Open Access This article is distributed under the terms of the Creative Commons Attribution 4.0 International License (http:// creativecommons.org/licenses/by/4.0/), which permits unrestricted use, distribution, and reproduction in any medium, provided you give appropriate credit to the original author(s) and the source, provide a link to the Creative Commons license, and indicate if changes were made.

Publisher's Note Springer Nature remains neutral with regard to jurisdictional claims in published maps and institutional affiliations.

\section{References}

1. HES data: Health and Social Care Information Centre. Hospital Episode Statistics. http://www.hesonline.nhs.uk/Ease/servlet/ ContentServer?siteID=1937. Accessed 11June 2018

2. Kirby A, Burnside G, Bretsztajn L, Burke D (2015) Post-operative infections following colorectal surgery in an English teaching hospital. Infect Dis (Lond) 47:825-829

3. Petrosillo N, Drapeau CM, Nicastri E, Martini L, Ippolito G, Moro ML (2008) Surgical site infections in Italian hospitals: a prospective multicenter study. BMC Infect Dis 8:4

4. Smith RL, Bohl JK, Mcelearney ST, Friel CM, Barclay MM, Sawyer RG, Foley EF (2004) Wound infection after elective colorectal resection. Ann Surg 239:599-605

5. Wick EC, Gibbs L, Indorf LA, Varma MG, Garcia-Aguilar J (2008) Implementation of quality measures to reduce surgical site infection in colorectal patients. Dis Colon Rectum 51:1004-1009

6. Tanner J, Khan D, Aplin C, Ball J, Thomas M, Bankart J (2009) Post-discharge surveillance to identify colorectal surgical site infection rates and related costs. J Hosp Infect 72:243-250

7. Jenks PJ, Laurent M, McQuarry S, Watkins R (2014) Clinical and economic burden of surgical site infection (SSI) and predicted financial consequences of elimination of SSI from an English hospital. J Hosp Infect 86:24-33

8. Nelson RL, Gladman E, Barbateskovic M (2014) Antimicrobial prophylaxis for colorectal surgery. Cochrane Database Syst Rev 5:CD001181

9. Itani KM, Jensen EH, Finn TS, Tomassini JE, Abramson MA (2008) Effect of body mass index and ertapenem versus cefotetan prophylaxis on surgical site infection in elective colorectal surgery. Surg Infect 9:131-137

10. Teillant A, Gandra S, Barter D, Morgan DJ, Laxminarayan R (2015) Potential burden of antibiotic resistance on surgery and cancer chemotherapy antibiotic prophylaxis in the USA: a literature review and modelling study. Lancet Infect Dis 15:1429-1437

11. Kirby A, Bretsztajn L, Santoni N, Patel H, Burke D, Horner C (2015) Microbiological prediction of surgical site infection risk after colorectal surgery: a feasibility study. J Hosp Infect 90:271-272
12. Asín-Prieto E, Soraluce A, Trocóniz IF, Campo Cimarras E, Sáenz de Ugarte Sobrón J, Rodríguez-Gascón A, Isla A (2015) Population pharmacokinetic models for cefuroxime and metronidazole used in combination as prophylactic agents in colorectal surgery: modelbased evaluation of standard dosing regimens. Int J Antimicrob Agents 45:504-511

13. MacGowan AP (2011) Revisiting Beta-lactams - PK/PD improves dosing of old antibiotics. Curr Opin Pharmacol 11:470-476

14. Nascimento JW, Carmona MJ, Strabelli TM, Auler JO Jr, Santos SR (2007) Perioperative cefuroxime pharmacokinetics in cardiac surgery. Clinics (Sao Paulo) 62:257-260

15. Levey AS, Stevens LA, Schmid CH, Zhang YL, Castro AF 3rd, Feldman HI, Kusek JW, Eggers P, Van Lente F, Greene T, Coresh J, CKD-EPI (Chronic Kidney Disease Epidemiology Collaboration) (2009) A new equation to estimate glomerular filtration rate. Ann Intern Med 150:604-612

16. Ogungbenro K, Aarons L (2008) How many subjects are necessary for population pharmacokinetic experiments? Confidence interval approach. Eur J Clin Pharmacol 64:705-713

17. Mangram A, Horan TC, Pearson ML, Silver L, Jarvis WR (1999) Guideline for prevention of surgical site infection. Am J Infect Control 27:97-132

18. Public Health England. Surveillance of surgical site infections in NHS hospitals in England, 2016 to 2017. Public health England, December 2017. Available from: www.gov.uk/phe. Accessed 11 June 2017

19. Health Protection Agency (2012) English national point prevalence survey on healthcare associated infections and antimicrobial use, 2011: preliminary data HPA, London

20. Moher D, Hopewell S, Schulz KF, Montori V, Gøtzsche PC, Devereaux PJ, Elbourne D, Egger M, Altman DG (2010) CONSORT 2010 explanation and elaboration: updated guidelines for reporting parallel group randomised trials. BMJ 340:c869

21. Eldridge SM, Chan CL, Campbell MJ, Bond CM, Hopewell S, Thabane L, Lancaster GA, PAFS consensus group (2016) CONSORT 2010 statement: extension to randomised pilot and feasibility trials. BMJ 355:i5239

22. (EUCAST) ECoAST. Cefuroxime: rationale for the EUCAST clinical breakpoints (2010) http://www.eucast.org/fileadmin/src/media/ PDFs/EUCAST_files/Rationale_documents/Cefuroxime_iv Rationale_Document_1.0_2010Nov.pdf. Accessed 11 June 2018

23. Lovering AM, Perez J, Bowker KE, Reeves DS, MacGowan AP, Bannister G (1997) A comparison of the penetration of cefuroxime and cephamandole into bone, fat and haematoma fluid in patients undergoing total hip replacement. J Antimicrob Chemother 40:99104

24. Zelenitsky SA, Ariano RE, Harding GK, Silverman RE (2002) Antibiotic pharmacodynamics in surgical prophylaxis: an association between intraoperative antibiotic concentrations and efficacy. Antimicrob Agents Chemother 46:3026-3030

25. Woodfield JC, Beshay N, van Rij AM (2009) A meta-analysis of randomized, controlled trials assessing the prophylactic use of ceftriaxone. A study of wound, chest, and urinary infections. World J Surg 33:2538-2550

26. Shogan BD, Belogortseva N, Luong PM, Zaborin A, Lax S, Bethel C, Ward M, Muldoon JP, Singer M, An G, Umanskiy K, Konda V, Shakhsheer B, Luo J, Klabbers R, Hancock LE, Gilbert J, Zaborina O, Alverdy JC (2015) Collagen degradation and MMP9 activation by Enterococcus faecalis contribute to intestinal anastomotic leak. Sci Transl Med 7:286ra68 